\title{
Wandering spleen: a unique cause of acute abdomen
}

\author{
H. Hui Lian'1, F. Hayati ${ }^{1}$, A.A. Ali², N. Azizan³ , M.F. Che Ani' ${ }^{4}$, M.A. Suhaili², A.D. Zakaria ${ }^{5}$ \\ ${ }^{1}$ Department of Surgery, Faculty of Medicine and Health Sciences, Universiti Malaysia Sabah, Malaysia \\ ${ }^{2}$ Department of Surgery, Faculty of Medicine, Universiti Kebangsaan Malaysia, Malaysia \\ ${ }^{3}$ Department of Pathobiology and Medical Diagnostic, Faculty of Medicine and Health Sciences, \\ Universiti Malaysia Sabah, Malaysia \\ ${ }^{4}$ Department of General Surgery, Surgical Sciences Cluster, Faculty of Medicine, Universiti Teknologi MARA, Malaysia \\ ${ }^{5}$ Department of Surgery, School of Medical Sciences, Universiti Sains Malaysia, Malaysia
}

[Received: 1 September 2017; Accepted: 15 October 2017]

\begin{abstract}
We present the case of a 16-year-old boy presented with 2-week episode of worsening lower abdominal pain. Clinically, there was a tender palpable mass on the suprapubic region. Ultrasonography showed an absent spleen at its usual area, instead suprapubic mass suggestive of ectopic spleen was identified. An emergency laparotomy revealed a congested spleen in the pelvic cavity. Splenectomy was undertaken as it was non-viable. The patient was discharged uneventfully with triple vaccinations. We describe this unique entity with its literature review. (Folia Morphol 2018; 77, 2: 400-402)
\end{abstract}

Key words: wandering spleen, pelvic spleen, torsion, ultrasound

\section{INTRODUCTION}

Wandering or ectopic spleen is a rarely diagnosed clinical entity, with the incidence of less than $0.2 \%$ [6]. Congenital and acquired causes have been advocated to explain its onset. However, the precise aetiology is never completely understood due to rarity of the condition. The nature of the illness is only recognised when complications have occurred and often diagnosed in an emergency setting. Ectopic spleen is mostly congenital and described in the paediatric population, typically between ages 3 months and 10 years [5]. Acquired causes on the other hand include splenomegaly, trauma, ligamentous laxity and hormonal deficiencies secondary to pregnancy which makes ectopic spleen more frequently seen in females of reproductive age. Both congenital and acquired conditions result in a long pedicle, inclining to torsion and resultant partial or complete splenic infarction. Herein, we describe a unique case of wandering spleen who presented with acute abdomen.

\section{CASE REPORT}

A 16-year-old boy presented with lower abdominal pain for 2 weeks, which worsened for the past 3 days. He denied trauma or fall prior to this episode. There were no fever, change of bowel habit, constitutional symptoms, and anaemic symptoms. He was slightly dehydrated with blood pressure of $103 / 64 \mathrm{mmHg}$ and pulse rate of $90 \mathrm{bpm}$. There was a palpable mass over the suprapubic region measuring around $5 \times 5 \mathrm{~cm}$ in size, able to get above and below, mobile in four directions. It was mildly tender with dullness on percussion. However, there was resonance upon percussion on the throb space. Both testes were palpable. Other systemic examinations were unremarkable.

Address for correspondence: Dr. F. Hayati, Department of Surgery, Faculty of Medicine and Health Sciences, Universiti Malaysia Sabah, Malaysia, tel: 088-320000 ext. 611029, e-mail: firdaushayati@gmail.com 


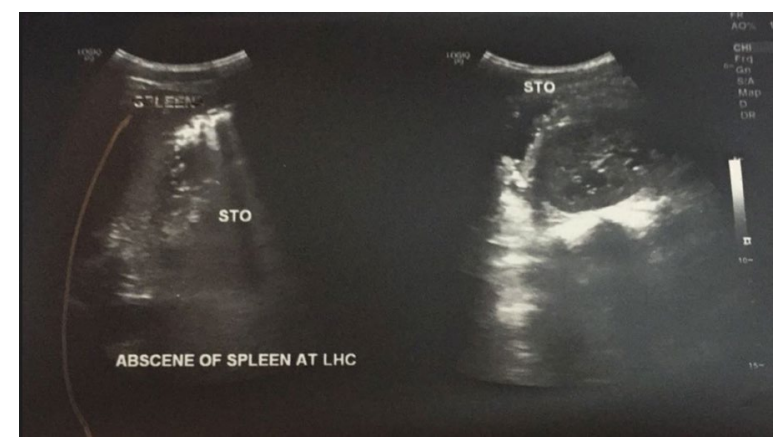

Figure 1. Ultrasonography showed absence of a spleen at left hypochondrial region; STO — stomach.

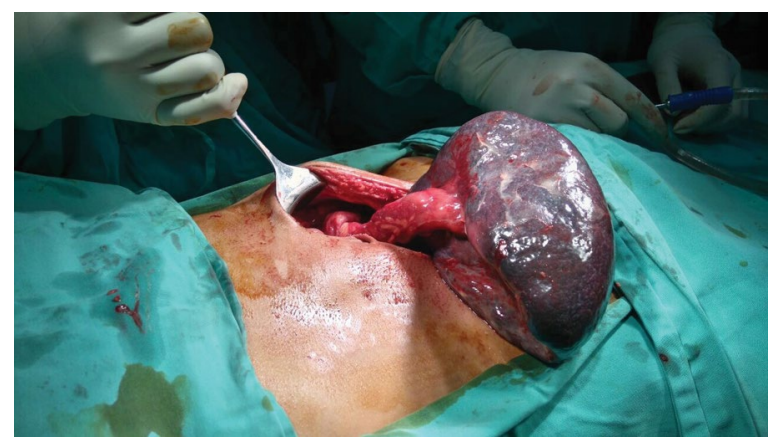

Figure 2. A congested spleen was located in the pelvic cavity with a long pedicle after derotation.

Investigations showed leukocytosis with total white cells count of $26.0 \times 10^{9} / \mathrm{L}$. Other investigations were normal. Abdominal ultrasound showed absence of spleen at its usual site, however a homogenous, hyperechoic ovoid mass measuring $7.2 \times 13.5 \mathrm{~cm}$ was identified in the pelvic region which was suggestive of ectopic spleen (Fig. 1). In view of reduced Doppler flow within, the possibility of incarcerated spleen cannot be ruled out. Other intraabdominal organs appeared normal.

An emergency laparotomy was performed. Intraoperatively, the spleen was identified in the pelvic cavity; it appeared congested. The pedicle was abnormally elongated and twisted 720 degree clockwise (Fig. 2). After adhesiolysis, a counterclockwise derotation was performed. Despite $100 \%$ oxygen and warm gauze wrap, the spleen continued to be non-viable. Decision for splenectomy was undertaken. The postoperative course was uneventful and the patient was discharged on the third postoperative day without any complication. The patient was then given vaccinations against Pneumo- coccus, Meningococcus and Haemophilus influenza 2 weeks after surgery. The histology of the resected spleen revealed congested splenic tissue with marked expansion of red pulp and reduced white pulp. No evidence of malignancy was seen.

\section{DISCUSSION}

Spleen is the largest organ in the reticulo-endothelial system. A normal spleen typically weighs from $150 \mathrm{~g}$ to $200 \mathrm{~g}$, with the length of $10.9 \pm 1.4 \mathrm{~cm}$, depth of $4.0 \pm 0.45 \mathrm{~cm}$ and diameter of $6.8 \pm$ $\pm 0.71 \mathrm{~cm} \mathrm{[1]}$. The normal spleen is located in the left hypochondrial region of the abdomen and is fixed in the intraperitoneal region just beneath the $9^{\text {th }}$ to $11^{\text {th }}$ intercostal spaces by five ligaments, namely lienorenal, splenocolic, splenophrenic, gastrosplenic and phrenicocolic ligaments [7]. In majority of the population, the spleen's convex area lies adjacent to the diaphragm and the concave side is in contact with the stomach, left kidney and splenic flexure. The hilum is located within this concavity and harbours the arterial, venous and lymphatic vessels and nerves. Though, it has been described in year 1972 that the "upside-down spleen" is also a normal variant due to an abnormal splenic rotation, rendering the hilum superiorly located and the convex border medial and adjacent to the left kidney [2]. Occasionally, this condition can be mistaken for a left suprarenal mass, giving another diagnostic dilemma [4]. Accessory spleen is, in fact, another variant of norm reported in $10-12 \%$ of population [3].

In adulthood, the presentations vary from splenic incidentaloma to acute abdominal emergency or chronic gastrointestinal complaints. The commonly described presentations are intermittent, recurrent abdominal pain due to splenic congestion with intermittent torsion of the splenic pedicle and its spontaneous detorsion [8]. In children, majority presented with acute surgical abdomen due to infarction from torsion of the splenic pedicle. The triad of a tender mobile mass with a notched edge, firmness in consistency and resonance upon percussion at the left upper quadrant are pathognomonic towards the diagnosis of a wandering spleen. Apart from tender pelvic mass, patient also presented with dysuria. The dysuria could be from the irritation of the ectopic spleen on the ureter.

Due to the unspecific clinical features of abdominal pain, imaging modalities play a crucial role in diagnosing ectopic spleen. These include ultrasonography, 
nuclear scintigraphy, contrast-enhanced computed tomography (CT) scanning, magnetic resonance imaging and angiogram [9]. Doppler ultrasound will guide the evaluation of splenic blood flow and organ viability. This is crucial since splenic decongestion and torsion can lead to an infarction. By performing a contrast-enhanced CT scan, findings of a wandering spleen include absence of spleen in the usual left upper quadrant, instead it will be localised elsewhere in the abdomen. Infarcted spleen will not have a contrast uptake and a whorled-pedicle of a spleen suggesting torsion can be visualised [2].

Surgery is the only definitive treatment for ectopic spleen in both complicated and uncomplicated cases. Without surgery, conservative treatment alone is associated with a complication rate as high as $65 \%$. Whether to perform splenopexy or splenectomy will depend on the intraoperative findings of a viable spleen. Splenectomy is indicated in splenomegaly, hypersplenism and torsion of the vascular pedicle with splenic infarction. Our patient presented with acute abdomen and splenic infarction, thus splenectomy was preferred to splenopexy by the operating surgeon to avoid any postoperative complications. For a viable spleen, splenopexy is the treatment of choice. Different techniques have been described: open or laparoscopic techniques and with or without using a mesh and peritoneal flaps [8].

\section{CONCLUSIONS}

The wandering spleen is a unique surgical entity. Simple ultrasonography enables clinician to diagnose such rare pathology, guided by the triad of abdominal signs for a wandering spleen. Upon diagnosis, treatment is usually surgical - either splenopexy or splenectomy, depending on the degree of torsion and splenic infarction.

\section{REFERENCES}

1. Badran DH, Kalbouneh HM, Al-Hadidi MT, et al. UItrasonographic assessment of splenic volume and its correlation with body parameters in a Jordanian population. Saudi Med J. 2015; 36(8): 967-972, doi: 10.15537/ smj.2015.8.11809, indexed in Pubmed: 26219448.

2. Cotes C, Swischuk LE. Left upper quadrant pain: upside down spleen. Pediatr Emerg Care. 2013; 29(9): 1009-1010, doi: 10.1097/PEC.0b013e3182a32079, indexed in Pubmed: 24201983.

3. d'Amico A, Cofalik A, Przeorek C, et al. Role of nuclear medicine imaging in differential diagnosis of accessory spleens in patients after splenectomy. Pol J Radiol. 2012; 77(1): 68-71, indexed in Pubmed: 22802870.

4. D'altorio RA, Cano JY. Upside-down spleen as cause of suprarenal mass. Urology. 1978; 11(4): 422-424, indexed in Pubmed: 664155.

5. Herman TE, Siegel MJ. CT of acute splenic torsion in children with wandering spleen. AJR Am J Roentgenol. 1991; 156(1): 151-153, doi: 10.2214/ajr.156.1.1898552, indexed in Pubmed: 1898552

6. Iqbal M, Tareen MA, Sohail U. Torsion of a wandering spleen presenting as a case of acute abdomen. J Coll Physicians Surg Pak. 2009; 19(2): 123-124, doi: 02.2009/ JCPSP.123124, indexed in Pubmed: 19208319.

7. Palas J, Matos AP, Ramalho M. The spleen revisited: an overview on magnetic resonance imaging. Radiol Res Pract. 2013; 2013: 219297, doi: 10.1155/2013/219297, indexed in Pubmed: 24377046.

8. Sayeed S, Koniaris L, Kovach S, et al. Torsion of a wandering spleen. Surgery. 2002; 132(3): 535-536, doi: 10.1067/ msy.2002.119494.

9. Zarroug $A E$, Hashim $Y$, El-Youssef $M$, et al. Wandering spleen as a cause of mesenteric and portal varices: a new etiology? J Pediatr Surg. 2013; 48(3): e1-e4, doi: 10.1016/j.jpedsurg.2012.12.042, indexed in Pubmed: 23480940. 